\title{
Reactive oxidants and myeloperoxidase and their involvement in neutrophil extracellular traps
}

\author{
Heather Parker* and Christine C. Winterbourn \\ Centre for Free Radical Research, Department of Pathology, University of Otago Christchurch, Christchurch, New Zealand
}

\section{Edited by:}

Marko Radic, University of Tennessee, USA

\section{Reviewed by:}

Nadine Varin-Blank, Institut National de la Santé et de Recherche

Médicale, France

Mariana J. Kaplan, University of

Michigan, USA

\section{*Correspondence:}

Heather Parker, Centre for Free Radical Research, Department of Pathology, University of Otago Christchurch, P.O. Box 4345,

Christchurch 8140, New Zealand. e-mail: heather.parker@otago.ac.nz
Neutrophils release extracellular traps (NETs) in response to a variety of inflammatory stimuli. These structures are composed of a network of chromatin strands associated with a variety of neutrophil-derived proteins including the enzyme myeloperoxidase (MPO). Studies into the mechanisms leading to the formation of NETs indicate a complex process that differs according to the stimulus. With some stimuli an active nicotinamide adenine dinucleotide phosphate (NADPH) oxidase is required. However, assigning specific reactive oxygen species involved downstream of the oxidase is a difficult task and definitive proof for any single oxidant is still lacking. Pharmacological inhibition of MPO and the use of MPO-deficient neutrophils indicate active MPO is required with phorbol myristate acetate as a stimulus but not necessarily with bacteria. Reactive oxidants and MPO may also play a role in NET-mediated microbial killing. MPO is present on NETs and maintains activity at this site. Therefore, MPO has the potential to generate reactive oxidants in close proximity to trapped microorganisms and thus effect microbial killing. This brief review discusses current evidence for the involvement of reactive oxidants and MPO in NET formation and their potential contribution to NET antimicrobial activity.

Keywords: superoxide, hydrogen peroxide, hypochlorous acid

\section{INTRODUCTION}

Neutrophils release extracellular traps (NETs) in response to a diverse range of stimuli including a variety of microorganisms, microbial products, and chemokines (refer to the review by Guimaraes-Costa et al., 2012 for a more detailed list). NETs are composed of a scaffold of chromatin decorated with an assortment of neutrophil-derived proteins, including the enzyme myeloperoxidase (MPO; Urban et al., 2009). NETs are believed to contribute to host defense, supplementary to neutrophil phagocytosis, by trapping and potentially killing invading pathogens (Brinkmann et al., 2004). However, extended exposure of self-DNA and damaging neutrophil granule proteins may be detrimental to the host and NETs have been linked with autoimmunity (Kessenbrock et al., 2009; Lande et al., 2011) and other pathological conditions (Clark et al., 2007; Fuchs et al., 2010; Narasaraju et al., 2011; Caudrillier et al., 2012).

Activated neutrophils produce large amounts of superoxide $\left(\mathrm{O}_{2}^{\bullet-}\right)$ via their nicotinamide adenine dinucleotide phosphate (NADPH) oxidase. $\mathrm{O}_{2}^{\bullet-}$ dismutates to hydrogen peroxide $\left(\mathrm{H}_{2} \mathrm{O}_{2}\right)$ leading to the formation of a variety of toxic oxygen derivatives, especially those formed by MPO-catalyzed reactions. Both the NADPH oxidase and MPO have been implicated in the regulation of NET formation. However, the specific reactive oxygen species (ROS) required remains to be clarified.

Myeloperoxidase catalyses the oxidation of chloride by $\mathrm{H}_{2} \mathrm{O}_{2}$ forming the strong oxidant hypochlorous acid $(\mathrm{HOCl})$, the prime mediator of oxidative killing in the phagosome (Winterbourn and Kettle, 2012). MPO is present on NETs (Urban et al., 2009) and has the potential (given a supply of $\mathrm{H}_{2} \mathrm{O}_{2}$ ) to generate $\mathrm{HOCl}$ in close proximity to trapped bacteria, thus providing a prospective mechanism for oxidative NET-mediated killing. In this short review, we summarize experimental evidence for the involvement of ROS and MPO in the regulation of NET formation and discuss their potential contribution to NET antimicrobial activity.

\section{ROS AND MPO IN NET FORMATION}

Studies into the mechanisms of NET formation (NETosis) indicate a complex process that differs depending on the stimulus. Given the variability in NET inducers (Guimaraes-Costa et al., 2012) the existence of more than one pathway is perhaps not surprising. The term NETosis is sometimes used to describe only those forms of NET formation associated with cell death (Steinberg and Grinstein, 2007), but NETs can be released from living cells (Yipp et al., 2012), and here we use NETosis to describe any form of NET formation. NETs differ with respect to composition, timing, the involvement of cell death and dependency on reactive oxidants (Clark et al., 2007; Fuchs et al., 2007; Yousefi et al., 2009; Pilsczek et al., 2010). To date, the majority of inducers examined show dependency on an active NADPH oxidase and there is evidence that with some stimuli MPO is also involved.

\section{NADPH OXIDASE DEPENDENCY}

Evidence that an active NADPH oxidase is required for NET formation has come from studies using inhibitors of the oxidase, knockout mice, or neutrophils from patients with chronic granulomatous disease (CGD) whose NADPH oxidase is nonfunctional (Stasia and $\mathrm{Li}, 2008$ ). Inhibition of the oxidase with diphenyleneiodonium chloride (DPI) prevents NETosis in response to several factors, including phorbol myristate acetate (PMA; Fuchs et al., 2007), an nitric oxide (NO) donor (Keshari et al., 2012), bacteria (Parker et al., 2012b), lipopolysaccharide (LPS; Yost et al., 2009), and complement factor 5a (C5a) after 
priming with granulocyte/macrophage colony-stimulating factor (GM-CSF; Yousefi et al., 2009). Interestingly with Staphylococcus aureus, an early phase of NET release induced by secreted bacterial products is independent of the oxidase and of cell death, with dependency on these increasing over time (Pilsczek et al., 2010). The later release of NETs was possibly induced by bacterial phagocytosis, which would have been slow under the conditions employed in this study. Thus, two different forms of NET stimulation could have operated over the course of the experiments. From this study it might be assumed that activation of the oxidase leads to NET expulsion by cell death and that the oxidase is not required for release from viable cells. However, oxidase-dependent NET release from living cells has been reported (Yousefi et al., 2009).

Strong evidence for NADPH oxidase-dependent NETosis comes from the finding that CGD neutrophils do not form NETs when stimulated with PMA, bacteria (Fuchs et al., 2007), or GM$\mathrm{CSF}+\mathrm{C} 5 \mathrm{a}$ (Yousefi et al., 2009). Exogenously added $\mathrm{H}_{2} \mathrm{O}_{2}$ restores the ability of CGD neutrophils to produce NETs (Fuchs et al., 2007), as does gene therapy to reconstitute NADPH oxidase function (Bianchi etal., 2009). Using a mouse model of CGD, Ermert et al. (2009) found that $g p 91^{-/-}$mice neutrophils do not make NETs when stimulated with PMA or Candida albicans. Furthermore, using genetically different inbred mouse strains these investigators observed that the level of NET formation correlated with the amount of ROS produced.

NET formation can also occur independently of oxidase activity. Not all stimulants activate the oxidase (Farley et al., 2012) and some that do may induce NETs independent of this. For example, the calcium ionophore ionomycin activates the NADPH oxidase yet induces NETs similarly in the presence or absence of DPI (Parker et al., 2012b). S. aureus leukocidins also induce NETs when oxidase activity is inhibited (Pilsczek et al., 2010). The oxidative burst was not measured in this study; however, similar concentrations of purified leukocidin combinations can induce ROS production (Colin and Monteil, 2003).

Although DPI is a general flavoenzyme inhibitor, the most likely explanation for its effect on NETosis is that it inhibits the NADPH oxidase, and this is supported by the CGD neutrophil and knockout mice studies. DPI does have other effects, including inhibition of mitochondrial complex I and inducible nitric oxide synthase (iNOS). However, even though an NO donor has been shown to induce NETs (Keshari et al., 2012), the low levels of iNOS in isolated human neutrophils make it unlikely that DPI prevents NETosis by inhibiting iNOS. Of note, a recent report describes DPI-sensitive NET induction by platelet activating factor, which does not activate the oxidase (Farley et al., 2012).

\section{THE ROLE OF MPO}

There is growing evidence that MPO is necessary for PMAstimulated NETosis and the majority of studies indicate that an active enzyme is required. Inhibition of MPO decreases PMAstimulated NETs (Akong-Moore et al., 2012; Palmer et al., 2012; Parker et al., 2012b) and neutrophils from MPO-deficient patients have reduced ability to produce NETs when stimulated with PMA. Metzler et al. (2011) found the level of NETs produced correlated with the degree of MPO deficiency and that neutrophils completely deficient in MPO could not make NETs. We observed just 3\% of normal MPO activity was sufficient to allow PMA-induced NETosis (Parker et al., 2012b). Inhibition of this residual activity abrogated NET formation (Figure 1A).

Myeloperoxidase may not be required with all stimuli. We found inhibiting MPO in control donor neutrophils had no effect on Pseudomonas aeruginosa, S. aureus, or Escherichia coli NET induction (Parker et al., 2012b). MPO-deficient neutrophils also made NETs as efficiently as those from control donors when stimulated with $P$. aeruginosa and inhibition of residual MPO activity had no effect (Figure 1B; Parker et al., 2012b). In contrast to our observations, Akong-Moore et al. (2012) prevented Pseudomonasinduced NETosis with MPO inhibition. Our conditions favored phagocytosis (Parker et al., 2012b) and may account for the differences observed between the studies but this remains to be explored. Interestingly, MPO inhibition or knock out had no effect on NETosis in mouse neutrophils (Akong-Moore et al., 2012) indicating an apparent species-specific difference in NET formation. Of note, mouse neutrophils contain less MPO than human (Rausch and Moore, 1975).

Myeloperoxidase is reported to contribute toward NETosis, independent of its activity, by aiding chromatin decondensation (Papayannopoulos et al., 2010). Purified MPO increased nuclear decondensation in a cell-free system but the most dramatic increase occurred when MPO was added in conjunction with neutrophil elastase. In PMA-stimulated neutrophils, elastase translocated to the nucleus early in NETosis while MPO localized there later, when NET release was occurring (Papayannopoulos et al., 2010). Therefore, in neutrophils MPO may not play a direct role in chromatin decondensation.

To sum up, there is good evidence that MPO is important for PMA induction of NETs. From our studies, it would appear that this is not the case with bacteria. However, there are inconsistencies in the results from different laboratories that require explanation. Whether MPO is required with other physiological NET inducers is currently unknown. Nevertheless when MPO is needed, it appears that very little is actually required to facilitate NETosis.

\section{ASSIGNING THE SPECIFIC ROS REQUIRED}

Activation of the neutrophil NADPH oxidase leads to the production of a variety of ROS. Assigning which are required for NETosis is not simple. The site of oxidase activation and degree of degranulation, which vary depending on the stimulus, affect the relative amounts of the different ROS produced as well as access to different cell constituents. With soluble stimuli, such as PMA, and non-phagocytosed particulate stimuli, activation largely occurs at the plasma membrane although some occurs at intracellular sites (reviewed in Bylund et al., 2010; Figure 1C). As yet these are not well characterized. During phagocytosis, activation mainly occurs at the phagosomal membrane (Winterbourn and Kettle, 2012), but electron microscope evidence shows that some also occurs elsewhere in the cell (Robinson, 2008; Figure 1D).

The NADPH oxidase removes electrons from cellular NADPH and transfers them across a membrane to oxygen, forming $\mathrm{O}_{2}^{\bullet-}$ in the extracellular environment, phagosome or a currently undefined intracellular compartment. $\mathrm{O}_{2}^{\bullet-}$ is membrane impermeable but rapidly dismutates to membrane permeable $\mathrm{H}_{2} \mathrm{O}_{2}$. Some of 


\section{A PMA}

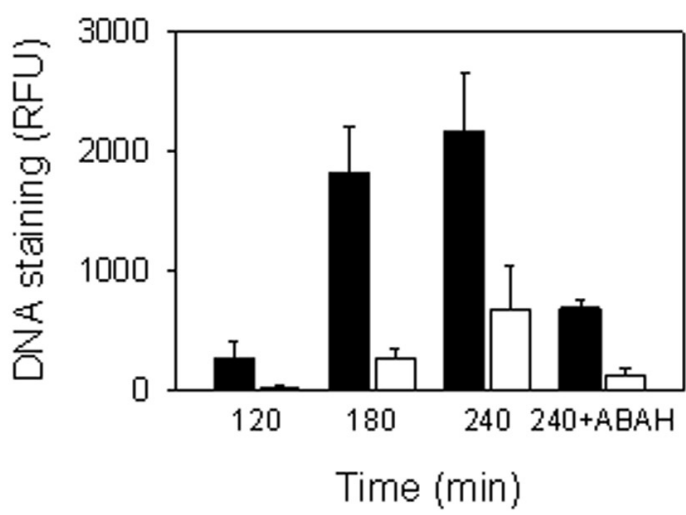

C

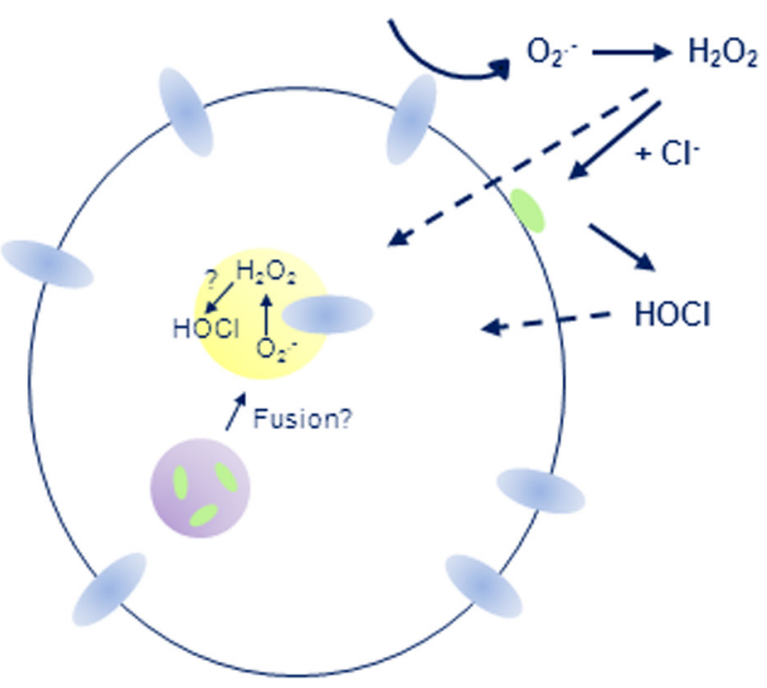

PMA \& non-phagocytic stimuli

FIGURE 1 | Myeloperoxidase (MPO) is required for PMA but not bacterial induction of NETs. (A,B) The release of NETs from control (filled bars) and MPO-deficient (open bars) neutrophils measured over $4 \mathrm{~h}$. MPO-deficient neutrophils formed NETs less efficiently with PMA, but not with $P$. aeruginosa, than neutrophils from control donors. To inhibit MPO, samples were incubated in the presence of $100 \mu \mathrm{M}$ of the MPO inhibitor 4-aminobenzoic acid hydrazide $(A B A H)$. Results are means \pm SEM of two to
B P. aenuginosa

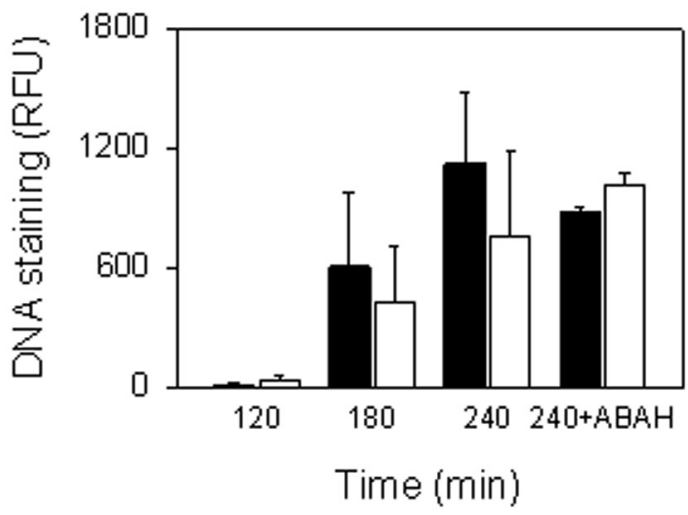

D

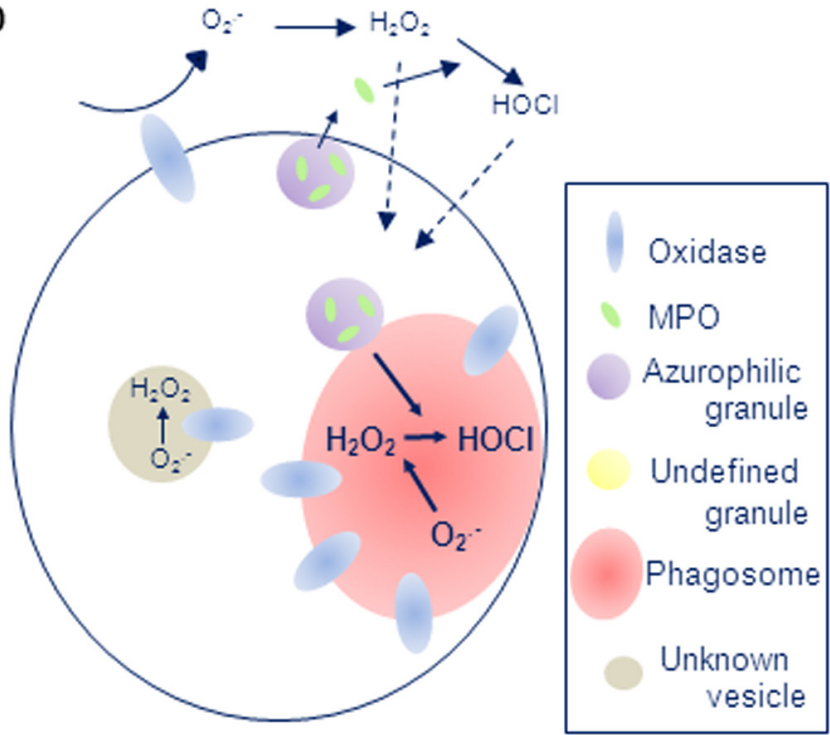

Phagocytosed stimuli

three independent experiments. For PMA, $p=0.02$ at $180 \mathrm{~min} ; p=0.071$ at 240 min by $t$-test. Data obtained with permission from Parker et al. (2012b). (C,D) Schematic representations of the intra- and extracellular locations of oxidant production in response to (C) soluble and non-phagocytic stimuli, or (D) phagocytosis (reviewed in Bylund et al., 2010 and Robinson, 2008). Details are given in the text. With PMA, oxidant production is predominately extracellular while phagocytosis induces largely intracellular production.

the $\mathrm{H}_{2} \mathrm{O}_{2}$ produced extracellularly may diffuse into the cell while some may react with MPO outside the cell (Figures 1 C,D). The production of $\mathrm{HOCl}$ in the extracellular environment requires MPO release, the timing or level of which varies with stimulus. In the phagosome, due to high MPO concentrations, essentially all of the $\mathrm{H}_{2} \mathrm{O}_{2}$ should react with MPO before it can diffuse out (Winterbourn and Kettle, 2012). $\mathrm{H}_{2} \mathrm{O}_{2}$ can also react to form hydroxyl radicals and singlet oxygen $\left({ }^{1} \mathrm{O}_{2}\right)$. However, the generation of these oxidants by neutrophils is considered to be very low (Winterbourn and Kettle, 2012). PMA gives a larger, more sustained oxidative burst than other stimulants that induce NETs.
However, even with PMA, oxidase activity is over well before NETs are released. $\mathrm{O}_{2}^{\bullet-}$ is produced within a minute of stimulation and continues for at least an hour but with the rate decreasing over this time (Decoursey and Ligeti, 2005). Similarly, oxidase activity continues for about $30 \mathrm{~min}$ following phagocytosis (Granfeldt and Dahlgren, 2001). Therefore, ROS produced must influence earlier rather than later events in NETosis.

By the nature of NADPH oxidase activation, it would seem it is likely that both the site of oxidant production and the nature of the oxidants produced are important in NET formation. Several groups have attempted to identify the specific ROS involved, 
primarily by using enzyme inhibitors or oxidant scavengers. One of the difficulties with this approach is targeting these to the appropriate compartment. It is straightforward to scavenge oxidants that are generated extracellularly. However, where there is intracellular oxidant production, as with PMA (Bylund et al., 2010), this is much more difficult to intercept. Consequently, there are still many uncertainties about what specific ROS generated by the NADPH oxidase or MPO are required in NETosis. The following sections discuss the evidence available for individual species.

\section{Hydrogen peroxide}

Several studies have shown that exogenously added $\mathrm{H}_{2} \mathrm{O}_{2}$ is sufficient to induce NETs (Fuchs etal., 2007; Neeli et al., 2009; Lim et al., 2011). However, addition of an oxidant and observation of NETs does not necessarily mean that this oxidant is responsible with physiological stimuli. With PMA, addition of catalase to scavenge extracellular $\mathrm{H}_{2} \mathrm{O}_{2}$ has little or no effect on NETosis (Fuchs etal., 2007; Parker et al., 2012b). It is plausible sufficient $\mathrm{H}_{2} \mathrm{O}_{2}$ is generated intracellularly to induce NETs so that extracellular scavenging would have minimal effect. This was examined using polyethylene glycol-catalase (PEG-catalase) which is taken up by endocytosis (Beckman et al., 1988), though its intracellular compartment is unknown. PEG-catalase reduced but did not completely inhibit PMA-NETosis while bacterial induction of NETs was unaffected (Parker et al., 2012b). Most likely PEG-catalase did not gain access to the appropriate intracellular sites to exert a full effect. Use of catalase inhibitors, such as azide or amino-triazole, has given inconsistent results (Fuchs et al., 2007; Palmer et al., 2012; Parker et al., 2012b). However, these also inhibit MPO, which complicates interpretation of effects.

\section{Superoxide}

Addition of superoxide dismutase (SOD) to neutrophils has been shown to modestly increase PMA-induced NETs (Palmer et al., 2012; Parker et al., 2012b). This would accelerate removal of extracellular $\mathrm{O}_{2}^{\bullet-}$ but have little effect on any generated intracellularly. Because most of the superoxide generated by neutrophils dismutates anyway, the presence of SOD would also make little difference to the amount of $\mathrm{H}_{2} \mathrm{O}_{2}$ produced (Winterbourn, 2008). At present we have no explanation for the SOD effect.

\section{Hypochlorous acid and other MPO products}

As the major strong oxidant produced by $\mathrm{MPO}, \mathrm{HOCl}$ is a potential candidate for the oxidant responsible for MPO-dependent NET formation. Indeed, addition of $\mathrm{HOCl}$ to neutrophils has been reported to induce NETosis (Akong-Moore et al., 2012; Palmer et al., 2012). However, there are issues with interpreting these results. First, in our experience $\mathrm{HOCl}$ concentrations $>50 \mu \mathrm{M}$ are rapidly toxic to neutrophils (Carr and Winterbourn, 1997), whereas the concentrations used to induce NETs were several millimolar. Second, $\mathrm{HOCl}$ was added to RPMI which contains numerous scavengers, including $>10 \mathrm{mM}$ amino acids, which would consume the $\mathrm{HOCl}$ within seconds (Pattison and Davies, 2006). Although this would overcome toxicity, it would mean that very little $\mathrm{HOCl}$ would reach the neutrophils. Many products including amino acid chloramines would be formed, but it

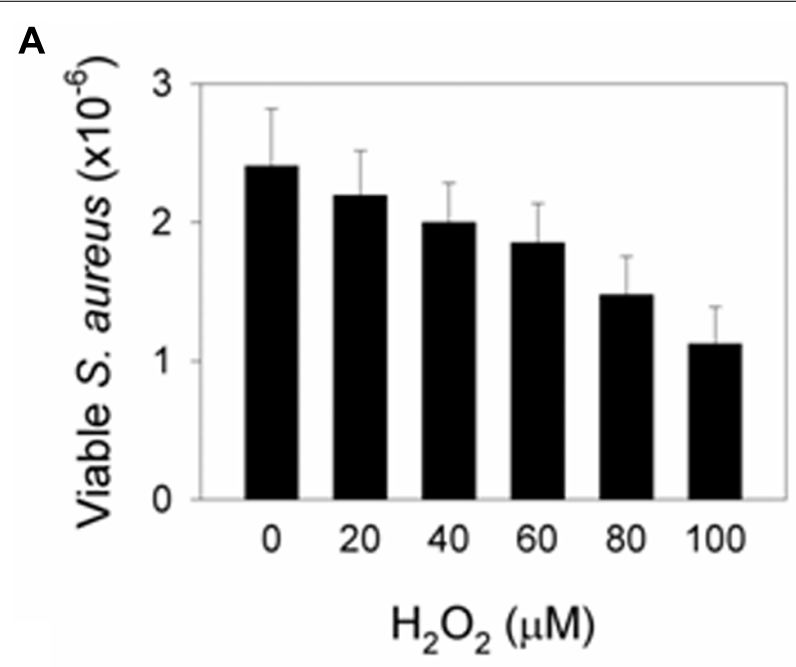

B

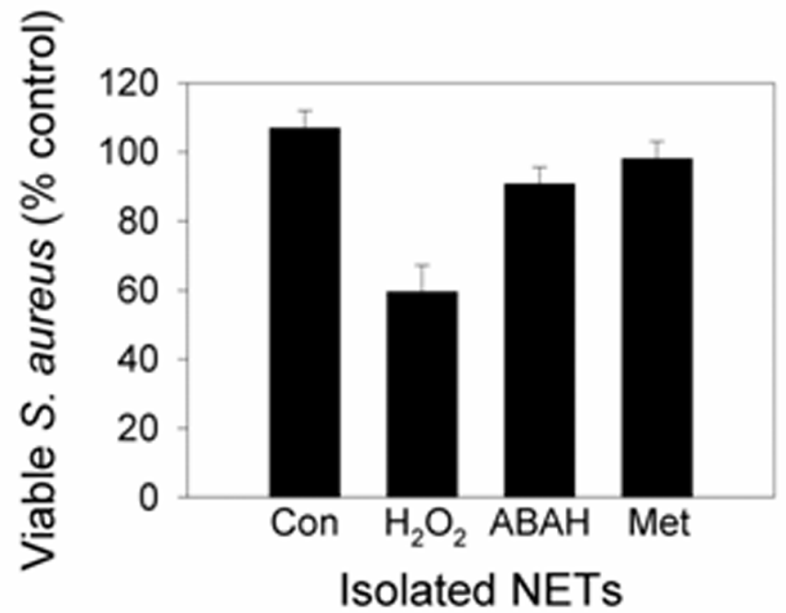

FIGURE 2 | Addition of $\mathrm{H}_{\mathbf{2}} \mathrm{O}_{\mathbf{2}}$ to NETs induces MPO-dependent killing. Neutrophils were stimulated with PMA to form NETs then incubated with $S$. aureus in the presence or absence of $(\mathbf{A})$ varying concentrations of $\mathrm{H}_{2} \mathrm{O}_{2}$ or (B) $100 \mu \mathrm{M} \mathrm{H}_{2} \mathrm{O}_{2}$ ladded in $20 \mu \mathrm{M}$ aliquots every 5 min to facilitate MPO turnover). At the examined concentrations, $\mathrm{H}_{2} \mathrm{O}_{2}$ in the absence of NETs had no significant effect on $S$. aureus viability. (A) Bacterial numbers significantly decreased with $\geq 40 \mu \mathrm{M} \mathrm{H}_{2} \mathrm{O}_{2}(p<0.05$, $t$-test on normalized data, $n=3$ ). (B) Bacterial viability decreased with $\mathrm{H}_{2} \mathrm{O}_{2}(p<0.001)$, and inhibition of MPO with $\mathrm{ABAH}$ and scavenging of $\mathrm{HOCl}$ with methionine (Met) prevented killing ( $p<0.01$; one-way ANOVA with Holm-Sidak pairwise comparison, $n=5)$. Results are presented as percent of control cells (Con) incubated with NETs alone. Data obtained with permission from Parker etal. (2012a).

is unclear which would be responsible for NET formation. Third, addition of catalase to prevent extracellular $\mathrm{HOCl}$ formation, or removing $\mathrm{HOCl}$ with the potent scavenger methionine, did not inhibit PMA-stimulated NET formation (Parker et al., 2012b). Inhibition by $>50 \mathrm{mM}$ taurine was seen (Palmer et al., 2012), but interpretation of this observation depends on the specificity of these high concentrations. It is still possible that $\mathrm{HOCl}$ generated intracellularly could be involved, but more definitive evidence is needed before drawing this conclusion. 
Alternative MPO products could be involved in NETosis. One example, singlet oxygen $\left({ }^{1} \mathrm{O}_{2}\right)$ has been implicated on the basis that NETs were observed after ${ }^{1} \mathrm{O}_{2}$ was generated using irradiated Photofrin (Nishinaka et al., 2011). However, while it is theoretically possible for neutrophils to generate ${ }^{1} \mathrm{O}_{2}$ from $\mathrm{H}_{2} \mathrm{O}_{2}$ and $\mathrm{HOCl}$ (Kiryu et al., 1999), it is a minor product (Hurst, 2012) and an unlikely candidate for NET regulation with other stimuli. MPO also catalyzes radical reactions, including lipid peroxidation. Interestingly, the radical scavenger Trolox inhibited PMA and LPS-induced NETosis in mouse neutrophils (Lim et al., 2011). This raises the possibility that a radical mechanism such as lipid peroxidation could be involved in the formation of NETs.

\section{Summary of ROS required}

In most cases, NADPH oxidase activity is needed for NET formation but the oxidants involved and their mechanisms of action are still unknown. The best, but not definitive, evidence is for $\mathrm{H}_{2} \mathrm{O}_{2}$ involvement, and with PMA a picture is emerging in which intracellularly generated MPO-derived ROS are important.

\section{INVOLVEMENT OF ROS AND MPO IN NET-MEDIATED MICROBIAL KILLING}

It has been postulated that the role of NETs in vivo is to trap and kill microorganisms and there are some excellent scanning electron micrographs of NETs entrapping both bacteria and fungi (Brinkmann et al., 2004; Beiter et al., 2006; Bruns et al., 2010). The evidence for direct killing by NETs is less convincing (Nauseef, 2012). Most studies have examined NET killing by incubating pre-formed NETs with bacteria then diluting and plating. In some instances, failure to release bacteria from NETs may have been interpreted as killing, a problem we encountered but overcame with DNase treatment to degrade NETs (Parker et al., 2012a). Using this method, several groups (Bruns et al., 2010; Menegazzi et al., 2012; Parker et al., 2012a) have observed that NETs on their own do not kill S. aureus, Aspergillus fumigatus conidia, or $C$. albicans blastospores.

\section{REFERENCES}

Akong-Moore, K., Chow, O. A., Von Kockritz-Blickwede, M., and Nizet, V. (2012). Influences of chloride and hypochlorite on neutrophil extracellular trap formation. PLoS ONE 7:e42984. doi: 10.1371/journal.pone. 0042984

Beckman, J. S., Minor, R. L. JR., White, C. W., Repine, J. E., Rosen, G. M., and Freeman, B. A. (1988). Superoxide dismutase and catalase conjugated to polyethylene glycol increases endothelial enzyme activity and oxidant resistance. J. Biol. Chem. 263, 6884-6892.

Beiter, K., Wartha, F., Albiger, B., Normark, S., Zychlinsky, A., and Henriques-Normark, B. (2006). An endonuclease allows Streptococcus pneumoniae to escape from neutrophil extracellular traps. Curr. Biol. $16,401-407$.

Bianchi, M., Hakkim, A., Brinkmann, V., Siler, U., Seger, R. A., Zychlinsky,
A., and Reichenbach, J. (2009). Restoration of NET formation by gene therapy in CGD controls aspergillosis. Blood 114, 2619-2622.

Brinkmann, V., Reichard, U., Goosmann, C., Fauler, B., Uhlemann, Y., Weiss, D. S., et al. (2004). Neutrophil extracellular traps kill bacteria. Science 303, 1532-1535.

Bruns, S., Kniemeyer, O., Hasenberg, M., Aimanianda, V., Nietzsche, S., Thywissen, A., etal. (2010). Production of extracellular traps against Aspergillus fumigatus in vitro and in infected lung tissue is dependent on invading neutrophils and influenced by hydrophobin RodA. PLoS Pathog. 6:e1000873. doi: 10.1371/journal.ppat.1000873

Bylund, J., Brown, K. L., Movitz, C., Dahlgren, C., and Karlsson, A. (2010). Intracellular generation of superoxide by the phagocyte NADPH oxidase: how, where, and what for? Free Radic. Biol. Med. 49, 1834-1845.

\section{EVIDENCE FOR MPO-MEDIATED NET KILLING}

Myeloperoxidase is present on NETs (Brinkmann et al., 2004; Urban etal., 2009; Parker etal., 2012a) placing it in close proximity to ensnared bacteria. NET-bound MPO is active and able to generate $\mathrm{HOCl}$ (Parker etal., 2012a). In our study, incubation of $S$. aureus with isolated NETs had no effect on bacterial viability. However, killing was observed when $\mathrm{H}_{2} \mathrm{O}_{2}$ was added as a substrate for MPO (Figure 2A). MPO inhibition and a potent $\mathrm{HOCl}$ scavenger prevented killing (Figure 2B). Therefore, NET-MPO has the potential to generate $\mathrm{HOCl}$ and effect microbial killing. At a site of inflammation, neutrophils that have formed NETs will no longer be producing ROS. However, during inflammation there is continued infiltration and activation of neutrophils which should provide the $\mathrm{H}_{2} \mathrm{O}_{2}$ required. The close proximity of NET-MPO to trapped microorganisms would be expected to facilitate exposure of microbes to lethal concentrations of $\mathrm{HOCl}$ and avoid all the oxidant being scavenged by the surrounding media. In vivo imaging using $\mathrm{HOCl}$ sensitive probes and differential fluorescent detection of live/dead bacteria would confirm if this occurs in living organisms.

\section{SUMMARY}

There is good evidence that the enzymatic processes of the NADPH oxidase and MPO are important in NETosis but elucidation of the specific ROS and their reactions that regulate NET formation requires further investigation. While the use of scavengers and inhibitors is a useful aid to the study of ROS in NET formation, interpretation of results is confounded by limitations of specificity and getting sufficient concentrations to intracellular locales where the critical oxidant generation may occur. The intracellular pathways leading to chromatin decondensation and NET release are still being worked out. Once this information becomes available, the involvement of oxidants in individual steps can be investigated and a clearer picture should emerge.

Carr, A. C., and Winterbourn, C. C. (1997). Oxidation of neutrophil glutathione and protein thiols by myeloperoxidase-derived hypochlorous acid. Biochem. J. 327( $\mathrm{Pt} 1)$, 275-281.

Caudrillier, A., Kessenbrock, K., Gilliss, B. M., Nguyen, J. X., Marques, M. B., Monestier, M., et al. (2012). Platelets induce neutrophil extracellular traps in transfusion-related acute lung injury. J. Clin. Invest. 122 , 2661-2671.

Clark, S. R., Ma, A. C., Tavener, S. A., Mcdonald, B., Goodarzi, Z., Kelly, M. M., et al. (2007) Platelet TLR4 activates neutrophil extracellular traps to ensnare bacteria in septic blood. Nat. Med. 13, 463-469.

Colin, D. A., and Monteil, H. (2003). Control of the oxidative burst of human neutrophils by staphylococcal leukotoxins. Infect. Immun. 71, 3724-3729.
Decoursey, T. E., and Ligeti, E. (2005). Regulation and termination of NADPH oxidase activity. Cell. Mol. Life Sci. 62, 2173-2193.

Ermert, D., Urban, C. F., Laube, B., Goosmann, C., Zychlinsky, A., and Brinkmann, V. (2009). Mouse neutrophil extracellular traps in microbial infections. J. Innate Immun. 1, 181-193.

Farley, K., Stolley, J. M., Zhao, P., Cooley, J., and Remold-O'Donnell, E. (2012). A SerpinB1 regulatory mechanism is essential for restricting neutrophil extracellular trap generation. J. Immunol. 189, 4574-4581.

Fuchs, T. A., Abed, U., Goosmann, C., Hurwitz, R., Schulze, I., Wahn, V., et al. (2007). Novel cell death program leads to neutrophil extracellular traps. J. Cell Biol. 176, 231-241.

Fuchs, T. A., Brill, A., Duerschmied, D., Schatzberg, D., Monestier, M., Myers, D. D. JR., etal. (2010). Extracellular DNA traps promote thrombosis. 
Proc. Natl. Acad. Sci. U.S.A. 107, 15880-15885.

Granfeldt, D., and Dahlgren, C. (2001). An intact cytoskeleton is required for prolonged respiratory burst activity during neutrophil phagocytosis. Inflammation 25, 165-169.

Guimaraes-Costa, A. B., Nascimento, M. T., Wardini, A. B., Pinto-Da-Silva, L. H., and Saraiva, E. M. (2012). ETosis: a microbicidal mechanism beyond cell death. J. Parasitol. Res. 2012, 929743.

Hurst, J. K. (2012). What really happens in the neutrophil phagosome? Free Radic. Biol. Med. 53, 508-520.

Keshari, R. S., Jyoti, A., Kumar, S., Dubey, M., Verma, A., Srinag, B. S. et al. (2012). Neutrophil extracellular traps contain mitochondrial as well as nuclear DNA and exhibit inflammatory potential. Cytometry A 81, 238-247.

Kessenbrock, K., Krumbholz, M., Schonermarck, U., Back, W., Gross, W. L., Werb, Z., et al. (2009). Netting neutrophils in autoimmune small-vessel vasculitis. Nat. Med. 15, 623-625.

Kiryu, C., Makiuchi, M., Miyazaki, J., Fujinaga, T., and Kakinuma, K. (1999). Physiological production of singlet molecular oxygen in the myeloperoxidase- $\mathrm{H}_{2} \mathrm{O}_{2}$-chloride system. FEBS Lett. 443, 154-158.

Lande, R., Ganguly, D., Facchinetti, V., Frasca, L., Conrad, C., Gregorio, J., et al. (2011). Neutrophils activate plasmacytoid dendritic cells by releasing self-DNA-peptide complexes in systemic lupus erythematosus. Sci. Transl. Med. 3, 73ra19.

Lim, M. B., Kuiper, J. W., Katchky, A., Goldberg, H., and Glogauer, M. (2011). Rac2 is required for the formation of neutrophil extracellular traps. J. Leukoc. Biol. 90, 771-776.

Menegazzi, R., Decleva, E., and Dri, P. (2012). Killing by neutrophil extracellular traps: fact or folklore? Blood $119,1214-1216$.
Metzler, K. D., Fuchs, T. A., Nauseef, W. M., Reumaux, D., Roesler, J., Schulze, I., et al. (2011). Myeloperoxidase is required for neutrophil extracellular trap formation: implications for innate immunity. Blood 117, 953-959.

Narasaraju, T., Yang, E., Samy, R. P., Ng, H. H., Poh, W. P., Liew, A. A., et al. (2011). Excessive neutrophils and neutrophil extracellular traps contribute to acute lung injury of influenza pneumonitis. Am. J. Pathol. 179, 199-210.

Nauseef, W. M. (2012). Editorial: Nye to NETs? A pause for healthy skepticism. J. Leukoc. Biol. 91, 353-355.

Neeli, I., Dwivedi, N., Khan, S., and Radic, M. (2009). Regulation of extracellular chromatin release from neutrophils. J. Innate Immun. 1 , 194-201.

Nishinaka, Y., Arai, T., Adachi, S., Takaori-Kondo, A., and Yamashita K. (2011). Singlet oxygen is essential for neutrophil extracellular trap formation. Biochem. Biophys. Res. Commun. 413, 75-79.

Palmer, L. J., Cooper, P. R., Ling, M R., Wright, H. J., Huissoon, A., and Chapple, I. L. (2012). Hypochlorous acid regulates neutrophil extracellular trap release in humans. Clin. Exp. Immunol. 167, 261-268.

Papayannopoulos, V., Metzler, K. D., Hakkim, A., and Zychlinsky, A. (2010). Neutrophil elastase and myeloperoxidase regulate the formation of neutrophil extracellular traps. J. Cell Biol. 191, 677-691.

Parker, H., Albrett, A. M., Kettle, A. J., and Winterbourn, C. C. (2012a). Myeloperoxidase associated with neutrophil extracellular traps is active and mediates bacterial killing in the presence of hydrogen peroxide. J. Leukoc. Biol. 91, 369-376.

Parker, H., Dragunow, M., Hampton, M. B., Kettle, A. J., and Winterbourn, C. C. (2012b). Requirements for NADPH oxidase and myeloperoxidase in neutrophil extracellular trap formation differ depending on the stimulus. J. Leukoc. Biol. $92,841-849$.

Pattison, D. I., and Davies, M. J. (2006) Reactions of myeloperoxidasederived oxidants with biological substrates: gaining chemical insight into human inflammatory diseases. Curr. Med. Chem. 13, 3271-3290.

Pilsczek, F. H., Salina, D., Poon, K. K., Fahey, C., Yipp, B. G., Sibley, C. D., et al. (2010). A novel mechanism of rapid nuclear neutrophil extracellular trap formation in response to Staphylococcus aureus. J. Immunol. 185, 7413-7425.

Rausch, P. G., and Moore, T. G. (1975). Granule enzymes of polymorphonuclear neutrophils: a phylogenetic comparison. Blood 46, 913-919.

Robinson, J. M. (2008). Reactive oxygen species in phagocytic leukocytes. Histochem. Cell Biol. 130, 281-297.

Stasia, M. J., and Li, X. J. (2008). Genetics and immunopathology of chronic granulomatous disease. Semin. Immunopathol. 30, 209-235.

Steinberg, B. E., and Grinstein, S. (2007). Unconventional roles of the NADPH oxidase: signaling, ion homeostasis, and cell death. Sci. STKE 2007, pe11.

Urban, C. F., Ermert, D., Schmid, M., Abu-Abed, U., Goosmann, C., Nacken, W., etal. (2009). Neutrophil extracellular traps contain calprotectin, a cytosolic protein complex involved in host defense against Candida albicans. PLoS Pathog. 5:e1000639. doi: 10.1371/journal. ppat. 1000639

Winterbourn, C. C. (2008). Reconciling the chemistry and biology of reactive oxygen species. Nat. Chem. Biol. 4, 278-286.

Winterbourn, C. C., and Kettle, A. J. (2012). Redox reactions and microbial killing in the neutrophil phagosome. Antioxid. Redox Signal. doi:10.1089/ars.2012.4827

[Epub ahead of print].

Yipp, B. G., Petri, B., Salina, D., Jenne, C. N., Scott, B. N., Zbytnuik, L. D., et al. (2012). Infection-induced NETosis is a dynamic process involving neutrophil multitasking in vivo. Nat. Med. 18, 1386-1393.

Yost, C. C., Cody, M. J., Harris, E. S., Thornton, N. L., Mcinturff, A. M., Martinez, M. L., et al. (2009). Impaired neutrophil extracellular trap (NET) formation: a novel innate immune deficiency of human neonates. Blood 113, 64196427.

Yousefi, S., Mihalache, C., Kozlowski, E., Schmid, I., and Simon, H. U. (2009). Viable neutrophils release mitochondrial DNA to form neutrophil extracellular traps. Cell Death Differ. 16, 1438-1444.

Conflict of Interest Statement: The authors declare that the research was conducted in the absence of any commercial or financial relationships that could be construed as a potential conflict of interest.

Received: 10 October 2012; accepted: 23 December 2012; published online: 21 January 2013.

Citation: Parker H and Winterbourn CC (2013) Reactive oxidants and myeloperoxidase and their involvement in neutrophil extracellular traps. Front. Immun. 3:424. doi: 10.3389/fimmu.2012.00424

This article was submitted to Frontiers in Molecular Innate Immunity, a specialty of Frontiers in Immunology. Copyright (c) 2013 Parker and Winterbourn. This is an open-access article distributed under the terms of the Creative Commons Attribution License, which permits use, distribution and reproduction in other forums, provided the original authors and source are credited and subject to any copyright notices concerning any third-party graphics etc. 